\title{
Phonon driven proton transfer in crystals with short strong hydrogen bonds
}

\author{
F. Fontaine-Vive \\ Institut Laue Langevin, BP 156, 38042 Grenoble Cedex 9, France \\ and Radiation Reactors and Radionuclides Department, Faculty of Applied Sciences, \\ Delft University of Technology, Mekelweg 15, $2629 \mathrm{JB}$ Delft, The Netherlands \\ M. R. Johnson ${ }^{\text {a) }}$ \\ Institut Laue Langevin, BP 156, 38042 Grenoble Cedex 9, France \\ G. J. Kearley \\ Radiation Reactors and Radionuclides Department, Faculty of Applied Sciences, \\ Delft University of Technology, Mekelweg 15, 2629 JB Delft, The Netherlands \\ J. A. Cowan \\ SRS Daresbury Laboratory, Daresbury, Cheshire WS4 4AD, England \\ J. A. K. Howard \\ Department of Chemistry, University of Durham, Durham DH1 3LE, United Kingdom \\ S. F. Parker \\ ISIS facility, Rutherford Appleton Laboratory, Oxon, United Kingdom OX11 OQX
}

(Received 16 March 2006; accepted 28 April 2006; published online 16 June 2006)

Recent work on understanding why protons migrate with increasing temperature in short, strong hydrogen bonds is extended here to three more organic, crystalline systems. Inelastic neutron scattering and density functional theory based simulations are used to investigate structure, vibrations, and dynamics of these systems as functions of temperature. The mechanism determined in a previous work on urea phosphoric acid of low frequency vibrations stabilizing average crystal structures, in which the potential energy well of the hydrogen bond has its minimum shifted towards the center of the bond, is found to be valid here. The new feature of the $\mathrm{N}-\mathrm{H} \cdots \mathrm{O}$ hydrogen bonds studied in this work is that the proton is transferred from the donor atom to the acceptor atom. Molecular dynamics simulations show that in an intermediate temperature regime, in which the proton is not completely transferred, the proton is bistable, jumping from one side of the hydrogen bond to the other. In the case of 3,5-pyridine dicarboxylic acid, which has been studied in most detail, specific phonons are identified, which influence the potential energy surface of the proton in the short, strong hydrogen bond. ( $) 2006$ American Institute of Physics. [DOI: 10.1063/1.2206774]

\section{INTRODUCTION}

Hydrogen bonds exhibit a wide range of structural and dynamic characteristics and play a fundamental role in many aspects of molecular science. Whereas many hydrogen bonds can be thought of as giving rise to a modest perturbation of the properties of molecules, short, strong hydrogen bonds (SSHBs) constitute a much stronger perturbation, which can result in a unique physical or chemical behavior. SSHBs are thought to play a fundamental role in stabilizing the intermediate state of certain enzymatic reactions through a subtle modification of the local environment of the hydrogen bond. ${ }^{1,2}$ Other implications of SSHBs in inhibitor potency of active site $^{3}$ or in stabilization of the photoactive yellow protein ${ }^{4}$ have been shown. DNA is an example of hydrogen bonded molecular system which contains the genetic code of living beings. Coding depends on the base-pair sequences and also on minor chemical changes to the nucleosides, such

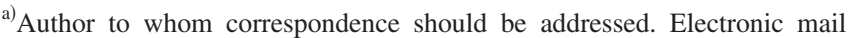
johnson@ill.fr
}

as the methylation of cytosine, which give rise to epigenetic effects. ${ }^{5}$ In this context, the temperature dependent dynamics of protons in DNA hydrogen bonds and the chemical integrity of the base-pair molecules are vital to the reliability of genetic coding.

The unique property of SSHBs of interest in this paper is the proton migration with increasing temperature away from the donor atom towards or beyond the center of the hydrogen bond. This effect has been observed by neutron diffraction in a number of compounds: urea phosphoric acid (UPA), ${ }^{6,7}$ pyridine-3,5-dicarboxylic acid (PDA), ${ }^{8,9} 1: 2$ cocrystal of benzene-1,2,4,5-tetracarboxylic acid and 4,4'-bipyridyl (BTA-BPY), ${ }^{10}$ and 1:1 crystal of 2-methylpyridien and pentachlorophenol (4-MP-PCP) $)^{11}$.

Temperature dependent proton migration can be thought of as being structurally or vibrationally driven. Lattice expansion could be sufficient to modify the geometry of the SSHB in an appropriate way, or there could be a subtle structural reorganization with temperature that favors a centered position of the proton in the hydrogen bond. Otherwise de- 
pending on the shape of the potential well of the hydrogen bond there could be excited vibrational states, which can be thermally populated, with a center of gravity shifted towards the center of the hydrogen bond.

Recent inelastic neutron scattering (INS) and $a b$ initio numerical simulations on UPA (Ref. 12) showed that vibrational levels in the potential well of the proton in the hydrogen bond could not be significantly populated at temperatures at which proton migration is observed. Total energy calculations based on density functional theory (DFT) methods showed the structures with a short donor-H distance to be most stable whatever the size of the unit cell is. Molecular dynamics (MD) with DFT revealed the proton migration effect. Analysis of the simulation trajectories showed low frequency lattice vibrations to be responsible for stabilizing the crystal structure at nonzero temperatures in such a way that the minimum of the average potential shifts towards the center of the hydrogen bond. The purpose of this paper is therefore to investigate whether the same mechanism drives proton migration in the other SSHBs.

UPA is a complicated system in that the crystal structure has many intermolecular hydrogen bonds, which involve all hydrogen atoms, and only one of which is short. As a result of this three-dimensional (3D) coupled network, particular phonons could not be identified as driving proton migration. The three other systems presented here have simpler hydrogen bond networks: two-dimensional (2D) sheets in the case of PDA, one-dimensional (1D) chains for BTA-BPY, and dimers for 4-MP-PCP. In contrast to UPA in which the SSHB is a $\mathrm{O}-\mathrm{H} \cdots \mathrm{O}$ bond, the three samples here present short $\mathrm{N}-\mathrm{H} \cdots \mathrm{O}$ bonds. In these three systems, there are fewer hydrogen bonds, which allow the hydrogen bond vibrations to be highlighted via selective deuteration in INS experiments. The three systems are also more pronounced examples of proton migration, the proton being transferred beyond the center of the hydrogen bond from donor to acceptor atoms. In this case does the vibrationally stabilized, time-averaged potential well take the proton continuously from donor to acceptor as a function of temperature?

In this paper we focus mainly on PDA for which we have used INS to investigate the molecular and hydrogen bond vibrations. These measurements are confronted with phonon calculations based on DFT methods, which allow the vibrational modes to be assigned, and mapping and anharmonic analyses of the hydrogen bond potential well. MD simulations using the same DFT methods allow temperature dependent effects to be investigated. These are not constrained to the low temperature crystal structure via the harmonic approximation, like thermal amplitudes determined from phonon calculations. For the other two samples, BTABPY and 4MP-PCP, only MD simulations have been performed to investigate directly proton migration. Experimental and computational details are described in the next section, and thereafter results are presented for each of the three systems.

\section{EXPERIMENTAL DETAILS}

PDA was obtained from Sigma-Aldrich and used in protonated form without further purification. The hydrogen bonds were deuterated by multiple recrystallizations from $\mathrm{D}_{2} \mathrm{O}$. INS spectra were measured at a temperature of $\sim 20 \mathrm{~K}$ on the inverted geometry spectrometers, IN1 at ILL (Ref. 13) and TOSCA at ISIS. ${ }^{14}$

\section{NUMERICAL DETAILS}

All DFT calculations were performed using the VASP DFT code. $^{15-18}$ We used projector augmented wave (PAW) pseudopotentials with a plane wave cutoff of $700 \mathrm{eV}$ in combination with the PBE functional. ${ }^{19}$ This energy cutoff is much higher than we normally use $(270 \mathrm{eV})$ and is found to give a significantly better description of the SSHBs. The $k$-point spacing was typically $0.1 \AA^{-1}$.

As for organic crystals ${ }^{20-24}$ and biopolymers, ${ }^{25}$ phonon calculations were performed for PDA using the direct method as implemented in the PHONON code. ${ }^{26} \mathrm{~A}$ supercell is constructed from the optimized unit cell structure, which allows all nonzero force constants to be determined when the crystallographically inequivalent atoms are displaced from their equilibrium positions. The force constants from VASP calculations are used to generate the dynamical matrix in PHONON, which is then diagonalized for a large number of randomly chosen points in the reciprocal lattice to obtain the vibrational density of states (vDOS). $S(Q, w)$ is calculated from the vDOS in the incoherent approximation for the specific geometry and resolution of each spectrometer.

Molecular dynamics simulations were also performed with VASP. The results presented here were obtained in the $N V E$ ensemble, that is, without coupling to an external heat bath. A time step of 1 fs was used, and the total simulation time was typically 5-10 ps. vDOSs were extracted from MD trajectories using NMOLDYN, ${ }^{27}$ and otherwise geometrical information was obtained directly from the time-dependent atomic coordinates.

The potential energy well for the proton in the hydrogen bond was determined for any given structure by calculating the crystal energy as a function of the displacement of the proton along the donor-acceptor direction. The energy levels within a potential well were determined from a numerical solution of Schrödinger's equation, assuming the mass of the oscillator to be that of the proton.

\section{RESULTS}

\section{A. Pyridine-3,5-dicarboxylic acid}

\section{Structure}

The structure of pyridine-3,5-dicarboxylic acid was reported at a number of temperatures in the range of $15-296 \mathrm{~K}$ by Cowan ${ }^{8}$ and Cowan et al. ${ }^{9}$ PDA crystallizes in the $P 2_{1} / c$ space group with four molecules in the unit cell. One short

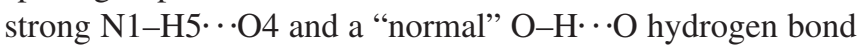
link the molecules in two-dimensional planar sheets in the $a-b$ plane.

Optimizing the crystal structure with VASP leaves the $a$ and $b$ axes almost unchanged but causes a significant expansion in the interplane, $c$ direction due to the underestimate of van der Waals (vdW) interactions in the DFT method. All simulations for PDA (and the other samples) have therefore 
TABLE I. Proton transfer as a function of temperature in the $\mathrm{N} 1 \cdots \mathrm{H} 5 \cdots \mathrm{O} 4$ SSHB in PDA as reported Refs. 8 and 9 and obtained from simulation.

\begin{tabular}{|c|c|c|c|c|}
\hline & $\mathrm{N} 1 \cdots \mathrm{O} 4$ & $\mathrm{~N} 1 \cdots \mathrm{H} 5$ & $\mathrm{H} 5 \cdots \mathrm{O} 4$ & $\mathrm{~N} 1-\mathrm{H} 5-\mathrm{O} 4$ \\
\hline \multicolumn{5}{|c|}{ Expt. data } \\
\hline $296 \mathrm{~K}$ & 2.525 & 1.308 & 1.218 & 177.9 \\
\hline $100 \mathrm{~K}$ & 2.526 & 1.181 & 1.361 & 172.3 \\
\hline $15 \mathrm{~K}$ & 2.523 & 1.213 & 1.311 & 176.4 \\
\hline \multicolumn{5}{|c|}{ MD simulations } \\
\hline $300 \mathrm{~K}$ & 2.541 & 1.206 & 1.331 & 169.3 \\
\hline $250 \mathrm{~K}$ & 2.546 & 1.162 & 1.371 & 170.1 \\
\hline $15 \mathrm{~K}$ & 2.535 & 1.128 & 1.409 & 174.2 \\
\hline \multicolumn{5}{|c|}{ Optimized geometries } \\
\hline $15 \mathrm{~K}$ & 2.551 & 1.122 & 1.429 & 176.5 \\
\hline
\end{tabular}

been performed in the experimentally determined unit cells. The short hydrogen bond geometry is reasonably well reproduced with a N-O length of $2.55 \AA$, a N-H length of $1.12 \AA$, and a $\mathrm{NHO}$ angle of $176.5^{\circ}$ compared to the corresponding experimental values of $2.523 \AA, 1.213 \AA$, and $176.4^{\circ}$ (see Table I). The calculated $\mathrm{N}-\mathrm{H}$ bond length is determined by the minimum in the potential energy surface, whereas the experimental value takes into account the zero point energy and the wave function of the hydrogen atom. Analyzing the energy levels in the potential well of the proton along the $\mathrm{N}-\mathrm{O}$ direction (see below) gives the underestimate of the $\mathrm{N}-\mathrm{H}$ bond length to be $0.03 \AA$. However, the normal $\mathrm{O}-\mathrm{H} \cdots \mathrm{O}$ hydrogen bond becomes shorter than the $\mathrm{N}-\mathrm{H} \cdots \mathrm{O}$ bond in terms of donor-acceptor distances, although the proton is tightly bound to the donor oxygen atom as indicated by the $\mathrm{O}-\mathrm{H}$ distance of $1.05 \AA$ (see Table II).

\section{Inelastic neutron scattering and phonon calculations}

The INS spectra for fully protonated PDA and a hydrogen bond deuterated sample (H4 and $\mathrm{H} 5$ in Fig. 1), both measured on TOSCA, are shown in Fig. 2 (upper curve in each panel). Also shown for each sample are the results of the corresponding VASP-PHONON calculations, with only the masses of the atoms changing in the construction of the corresponding dynamical matrices.

The main effect of deuterating the two hydrogen bonds in PDA is the pronounced reduction in intensity of the band

TABLE II. O1 $\cdots \mathrm{H} 4 \cdots \mathrm{O} 3$ hydrogen bond geometry as a function of temperature in PDA as reported in Refs. 8 and 9 and obtained from simulation.

\begin{tabular}{|c|c|c|c|c|}
\hline & $\mathrm{O} 1 \cdots \mathrm{O} 3$ & $\mathrm{O} 1 \cdots \mathrm{H} 4$ & $\mathrm{H} 4 \cdots \mathrm{O} 3$ & $\mathrm{O} 1-\mathrm{H} 4-\mathrm{O} 3$ \\
\hline \multicolumn{5}{|c|}{ Expt. data } \\
\hline $296 \mathrm{~K}$ & 2.591 & 1.004 & 1.608 & 165.1 \\
\hline $15 \mathrm{~K}$ & 2.569 & 1.025 & 1.558 & 167.7 \\
\hline \multicolumn{5}{|c|}{ MD simulations } \\
\hline $300 \mathrm{~K}$ & 2.564 & 1.037 & 1.546 & 164.2 \\
\hline $15 \mathrm{~K}$ & 2.516 & 1.049 & 1.471 & 172.1 \\
\hline \multicolumn{5}{|c|}{ Optimized geometries } \\
\hline $15 \mathrm{~K}$ & 2.471 & 1.091 & 1.383 & 175.3 \\
\hline
\end{tabular}

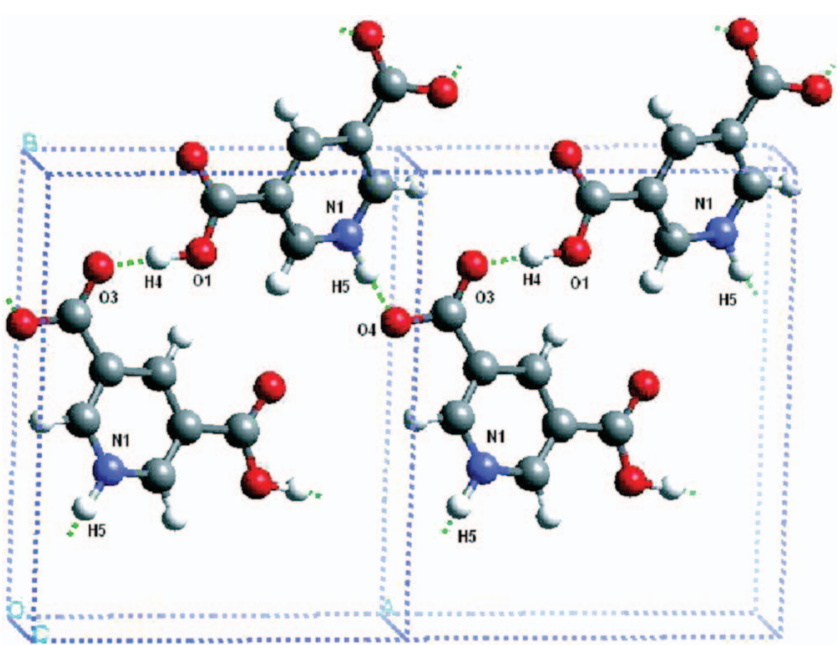

FIG. 1. (Color) An overall view of the low temperature crystal structure of PDA as reported in Refs. 8 and 9.

at $1400 \mathrm{~cm}^{-1}$. Inspection of the eigenvectors shows the band to represent a wagging motion of the $\mathrm{N}-\mathrm{H}$ and $\mathrm{O}-\mathrm{H}$ bonds. In the harmonic approximation, the stretching vibrations are found at the same frequency of $2200 \mathrm{~cm}^{-1}$ for the proton in both types of hydrogen bond in the calculation, but these are not observed in the INS spectra, even allowing for low intensity and poor statistics.

Mapping out the potential well of the proton in the $\mathrm{N}-\mathrm{H} \cdots \mathrm{O}$ bond shows a single-well, asymmetric form similar
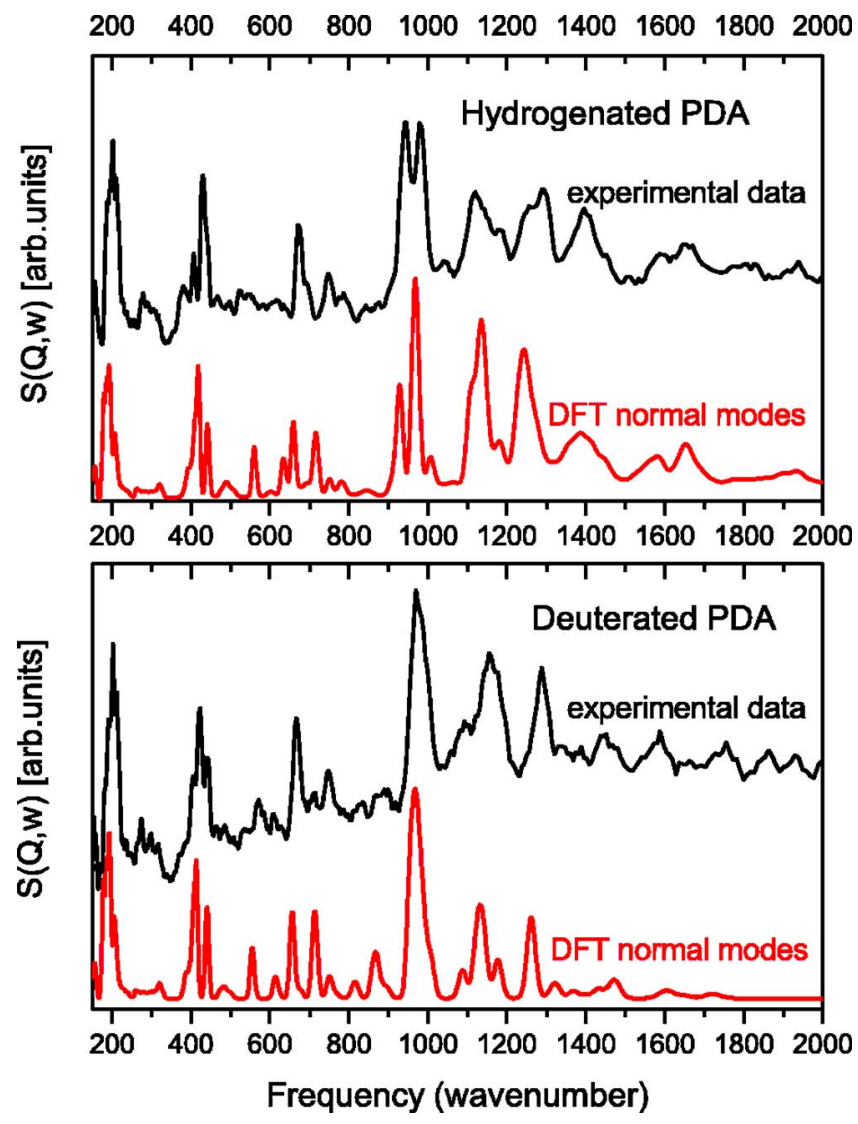

FIG. 2. Comparison of measured (upper curve) and calculated (lower curve, normal modes analysis) INS $S(Q, w)$ spectra of fully protonated (upper panel) and partially deuterated (lower panel)pyridine-3,5-dicarboxylic acid. 


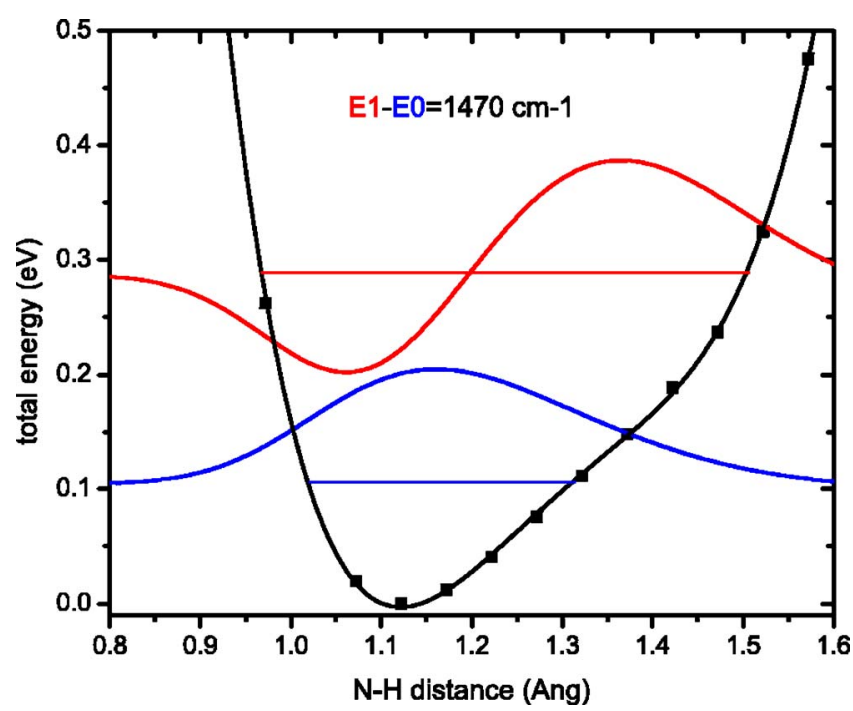

FIG. 3. Potential energy well, vibrational energy levels, and wave functions of the hydrogen atom in the SSHB of the optimized low temperature structure of PDA.

to the one determined for UPA (Fig. 3). Numerical solutions of Schrödinger's equation for a proton in this potential give a stretch-vibration frequency of $1470 \mathrm{~cm}^{-1}$. Allowing for a higher oscillator mass due to the coupling of such a low frequency, stretch vibration with other vibrational modes indicates that the main spectral change on deuteration also involves the stretching vibration.

\section{Molecular dynamics}

The forces needed to determine the acceleration of atoms in a MD simulation are derived in the same way as the forces needed in a phonon calculation. Thus having validated the DFT forces against INS experimental data, the MD simulation can be expected to describe accurately the solid state dynamics of PDA. MD simulations were performed at three temperatures: 15,250 , and $300 \mathrm{~K}$.

In view of the range of values discussed above for the $\mathrm{N}-\mathrm{H}$ stretch frequency in the SSHB, it is useful to consider the vDOS extracted from the MD simulations for the protons in the two hydrogen bonds (Fig. 4). Despite the hydrogen bonds having similar lengths in the optimized structure and at low temperature in the MD simulations (Tables I and II), their vDOSs behave very differently with increasing temperature. The $\mathrm{O}-\mathrm{H} \cdots \mathrm{O}$ bond assumes a geometry (Table II) and vDOS of a normal hydrogen bond as the temperature increases with the stretching mode moving to higher frequency. On the other hand, the SSHB shows the opposite behavior. The stretching band is found at $\sim 1800 \mathrm{~cm}^{-1}$ at the lowest temperature, while at $250 \mathrm{~K}$ and above, the band has merged with the wagging band at $1400 \mathrm{~cm}^{-1}$, as indicated by the foregoing analysis of the anharmonic potential well.

Extracting the donor-H distances from the MD trajectories (Table I and Fig. 5) underestimates the extent of proton transfer in the $\mathrm{N}-\mathrm{H} \cdots \mathrm{O}$ bond, as for UPA, the average proton position moving to the center of the hydrogen bond. However the time-dependent trace of the $\mathrm{N}-\mathrm{H}$ and $\mathrm{H} \cdots \mathrm{O}$ distances shows clearly that the proton is bistable, spending most of its time to the left or right of center and relatively

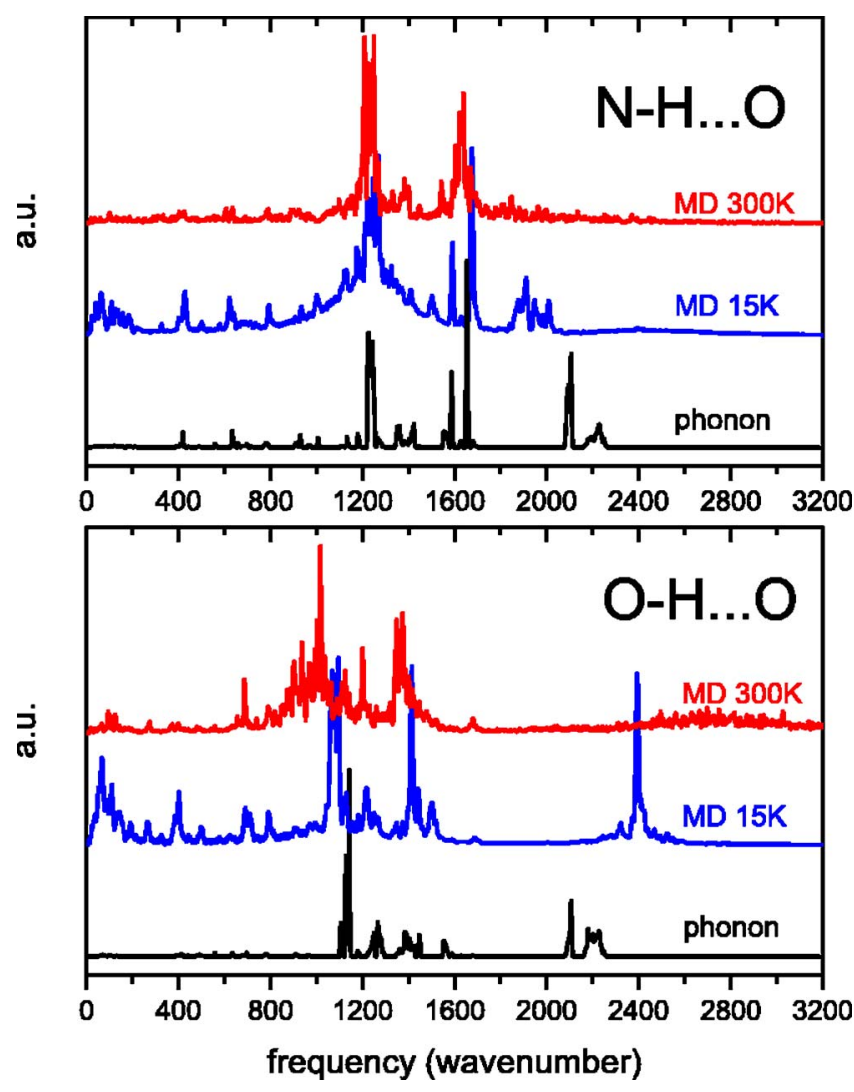

FIG. 4. Vibrational density of states of the protons in the short $\mathrm{N}-\mathrm{H} \cdots \mathrm{O}$ (upper panel) and "normal" $\mathrm{O}-\mathrm{H} \cdots \mathrm{O}$ (lower panel) hydrogen bond, determined from phonon calculations (lower curves) and from MD simulations at two temperatures (upper two curves).

little time at the center. This behavior is particularly evident from the histogram of the $\mathrm{N}-\mathrm{H}$ distance over the whole MD trajectory. In contrast, the $\mathrm{O}-\mathrm{H} \cdots \mathrm{O}$ bond, which the vDOS indicates to be a normal hydrogen bond at $250 \mathrm{~K}$, shows no proton migration, the proton remaining tightly bound to the donor $\mathrm{O}$ atom (Fig. 5, top panels).

Taking typical structures from the extrema of the $300 \mathrm{~K}$ trace in Fig. 5 and one for the centered position of the proton in the hydrogen bond allows the corresponding potential energy wells to be calculated (Fig. 6). For the extreme positions of the protons, asymmetric single wells are calculated. The centered proton position corresponds to a broad-bottomed potential well. The energy levels in these wells give rise to vibration frequencies ranging from $1200 \mathrm{~cm}^{-1}$ for the centered proton to $1500 \mathrm{~cm}^{-1}$ for the offset proton position, and therefore, at ambient temperature, the $\mathrm{N}-\mathrm{H}$ stretch vibration cannot drive proton migration. However the flat-bottomed potential well for the centered proton does allow the proton wave function to spread out and facilitate the passage of the proton from one side of the bond to the other.

The amplitude of the Fourier transform (FT) of the timedependent traces in Fig. 5 is shown in Fig. 7, which therefore shows the strength of the modulation of the $\mathrm{N}-\mathrm{H}$ distance as a function of vibration frequency. The most pronounced features are a low frequency band $\left(<50 \mathrm{~cm}^{-1}\right)$ and peaks at 115 and $145 \mathrm{~cm}^{-1}$. The phonon calculations show that the first three modes are acoustic phonons, two of which are polarized in the plane. The next three modes, up to $30 \mathrm{~cm}^{-1}$ at the 

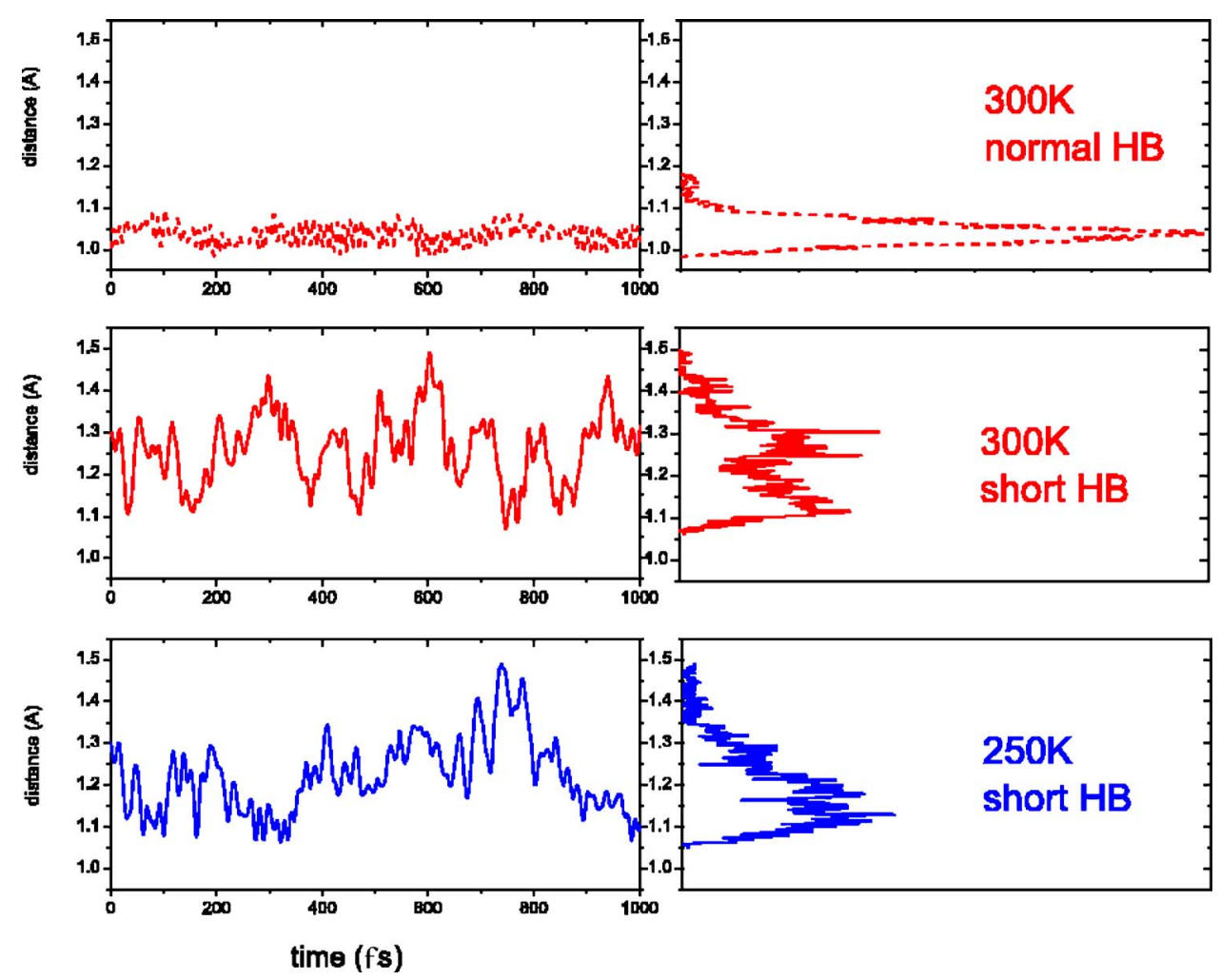

FIG. 5. N-H distance (lower two panels) and $\mathrm{O}-\mathrm{H}$ distance (upper panel) as a function of time over 1 ps. The histograms (right) are integrated over the whole MD trajectories.
$\Gamma$ point, are out of phase movements between the sheets, two of which are again polarized in the molecular planes. While at the $\Gamma$ point these infinitely long wavelength modes do not modulate the hydrogen bond geometry, this modulation can be achieved by shorter wavelength modes away from the $\Gamma$ point. These non- $\Gamma$-point modes would account for the first low frequency peak in the FT. The next in-plane vibrations occur at 125 and $155 \mathrm{~cm}^{-1}$, which, allowing for an anharmonic correction, match well the peaks observed at 115 and $145 \mathrm{~cm}^{-1}$ in the FT. Figure 8 shows the eigenvector of one of the modes at $155 \mathrm{~cm}^{-1}$, which clearly modulates the geometry of the hydrogen bond.

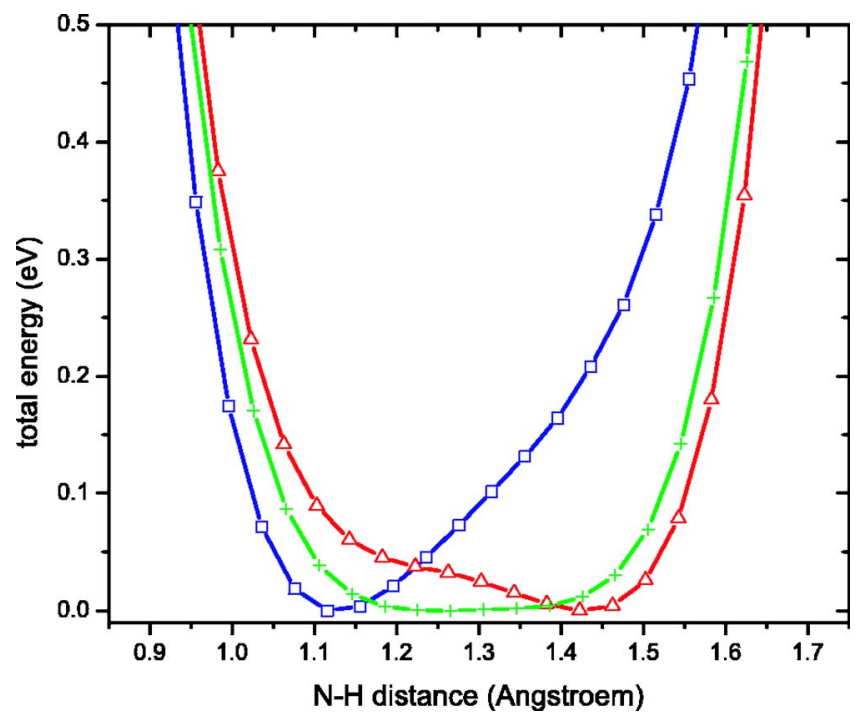

FIG. 6. Potential energy well of the hydrogen atom in the SSHB of structures from MD simulation. Open square symbols, structure with hydrogen atom close to N1. Open triangles, hydrogen atom close to O4. Crosses, hydrogen atom centered.
Partially deuterated PDA presents, experimentally, a more pronounced proton transfer effect, the smaller zero point motion tending to localize the proton nearer the donor or acceptor atom. As mentioned above, zero point motion is not included in our MD simulations and calculated proton transfer in the partially deuterated compound is very similar to that of the fully protonated compound (compare Tables I and III). Figures 5-8 are almost unchanged for partially deuterated PDA. While low frequency molecular and lattice vibrations are almost unchanged upon deuteration, this is not the case of the higher frequency modes involving the hydrogen bond protons. The foregoing vibrational analysis indi-

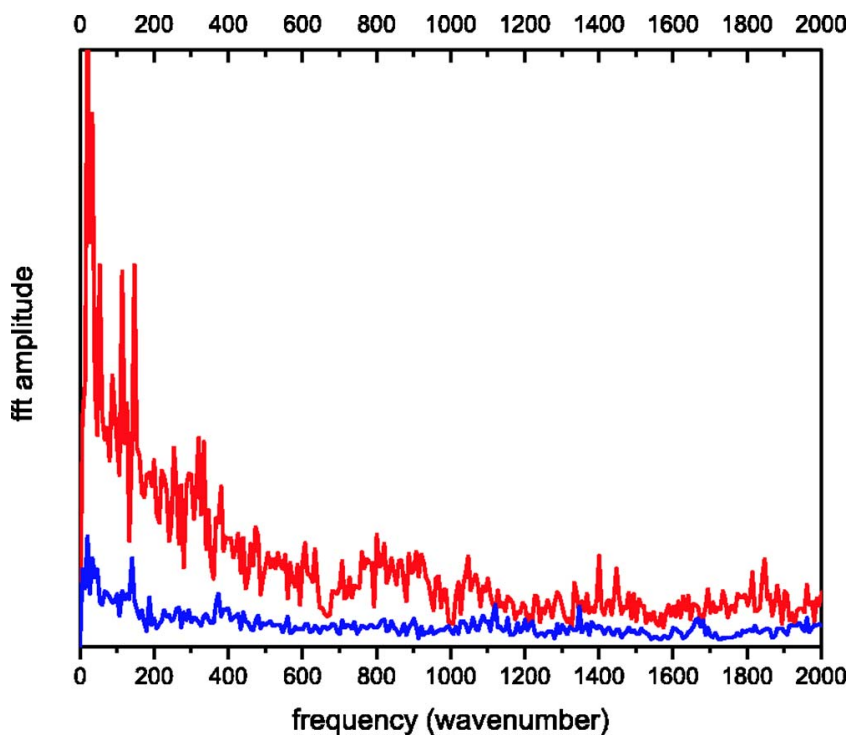

FIG. 7. The Fourier transform amplitude of the time-dependent N1-H5 bond length variation at $300 \mathrm{~K}$ (upper curve) and $15 \mathrm{~K}$ (lower curve). 


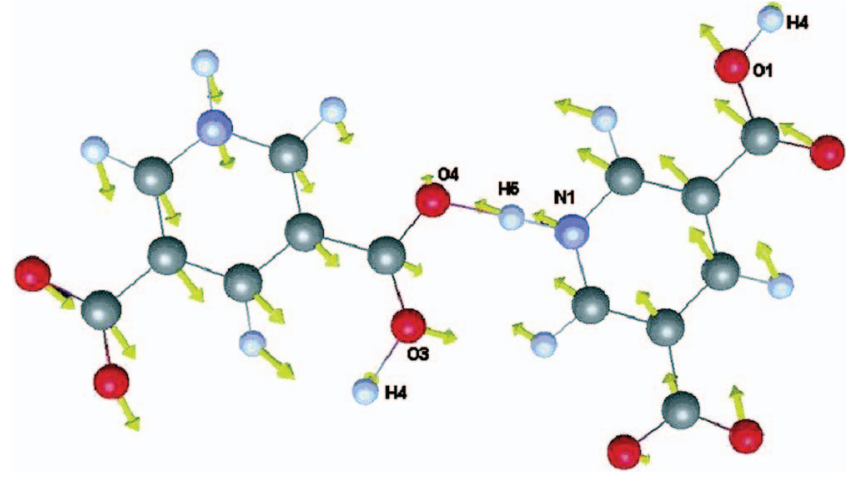

FIG. 8. (Color) Representation of the mode at $155 \mathrm{~cm}^{-1}$.

cates that stretching and wagging modes of the $\mathrm{N}-\mathrm{H}$ bond occur at $1400 \mathrm{~cm}^{-1}$ for $\mathrm{N}-\mathrm{H}$ and therefore at $\sim 990 \mathrm{~cm}^{-1}$ for $\mathrm{N}-\mathrm{D}$, contributing weakly to the strongest band in the INS spectra. If high frequency vibrations played a role in proton transfer, then the strong variation of these frequencies upon deuteration would lead to markedly different proton dynamics. That similar proton transfer effects are observed for protonated and deuterated compounds is further evidence of the dominant role of low frequency lattice dynamics.

\section{B. 1:2 cocrystal of benzene-1,2,4,5-tetracarboxylic acid and 4,4'-bipyridyl}

\section{Structural information}

The structure of the 1:2 cocrystal of benzene-1,2,4,5tetracarboxylic acid and 4,4'-bipyridyl was reported at number of temperatures in the range of 20-296 K by Cowan et $a l .{ }^{10}$ BTA-BPY crystallizes in a triclinic structure, in the $P$ -1 space group with two molecules in the unit cell. The SSHB N1-H1 $\cdots$ O22 is shown in Fig. 9 with atom labeling consistent with that of Cowan et al. The BTA and BPY molecules form hydrogen-bonded, 1D chains.

As with UPA and PDA the hydrogen bond length is overestimated by simple geometry optimization and the low temperature MD run (Table IV). Another characteristic of the DFT calculations is the donor-H distance which is shorter by $0.1 \AA$ than in the measurement. The evolution of the hydrogen bond geometry with temperature is very similar to that of PDA (compare Tables I and IV). The time dependence of the $\mathrm{N}-\mathrm{H}$ and $\mathrm{H} \cdots \mathrm{O}$ distances (Fig. 10) also hints at the same bistable behavior, which can be better detected by projecting the traces onto a histogram (Fig. 10).

The Fourier transform of the time-dependent traces in

TABLE III. Proton transfer as a function of temperature in the $\mathrm{N} 1 \cdots \mathrm{D} 5 \cdots \mathrm{O} 4 \mathrm{SSHB}$ in deuterated PDA as reported in Refs. 8 and 9.

\begin{tabular}{lcccc}
\hline \hline & $\mathrm{N} 1 \cdots \mathrm{O} 4$ & $\mathrm{~N} 1 \cdots \mathrm{D} 5$ & $\mathrm{D} 5 \cdots \mathrm{O} 4$ & $\mathrm{~N} 1-\mathrm{D} 5-\mathrm{O} 4$ \\
\hline Expt. data & & & & \\
$296 \mathrm{~K}$ & 2.564 & 1.457 & 1.108 & 177.6 \\
$15 \mathrm{~K}$ & 2.538 & 1.151 & 1.389 & 175.9 \\
$\begin{array}{l}\text { MD simulations } \\
\text { 300 K }\end{array}$ & & & \\
\hline \hline
\end{tabular}

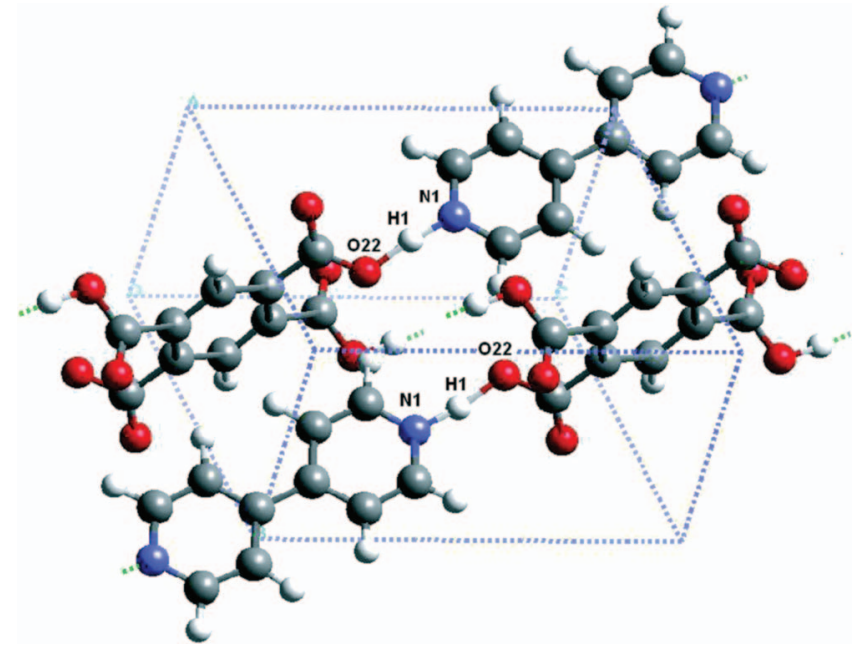

FIG. 9. (Color) Low temperature crystal structure of the 1:2 cocrystal of benzene-1,2,4,5-tetracarboxylic acid and 4,4'-bipyridyl as reported in Ref. 10.

Fig. 10 shows, as for PDA, that the low frequency phonons give rise to the strongest modulation of the $\mathrm{N}-\mathrm{H}$ bond length (Fig. 11), although, in this case, there are no well-defined frequencies.

\section{1:1 crystal of 4-methylpyridine and pentachlorophenol}

\section{Structural information}

The structure of the 1:1 crystal of 4-methylpyridine and pentachlorophenol was reported at low temperature by Steiner et al. ${ }^{11}$ 4-MP-PCP crystallizes in a triclinic structure, in the $P-1$ space group with two molecules in the unit cell. The SSHB O-H $\cdots \mathrm{N} 1$ is shown in Fig. 12 with atom labeling consistent with that of Steiner et al. The two molecules in this crystal form hydrogen-bonded dimers.

Table V shows that at low temperature the calculated hydrogen bond length is overestimated to a much greater extent $(0.1 \AA)$ than in the other samples. As a result there is little evolution in the hydrogen bond geometry as a function of temperature, the donor-acceptor distance shortening by $0.05 \AA$ which is mainly accommodated by a reduction in the proton-acceptor distance.

TABLE IV. Proton transfer as a function of temperature in the $\mathrm{N} 1 \cdots \mathrm{H} 1 \cdots \mathrm{O} 22$ SSHB in BTA-BPY as reported in Ref. 10 experimental data and simulation results.

\begin{tabular}{lcccc}
\hline \hline & $\mathrm{N} 1 \cdots \mathrm{O} 22$ & $\mathrm{~N} 1 \cdots \mathrm{H} 1$ & $\mathrm{H} 1 \cdots \mathrm{O} 22$ & $\mathrm{~N} 1-\mathrm{H} 1-\mathrm{O} 22$ \\
\hline Expt. data & & & & \\
$296 \mathrm{~K}$ & 2.531 & 1.302 & 1.241 & 169.6 \\
$200 \mathrm{~K}$ & 2.521 & 1.251 & 1.281 & 169.4 \\
$20 \mathrm{~K}$ & 2.522 & 1.207 & 1.325 & 170.2 \\
\multicolumn{2}{l}{ MD simulations } & & & \\
$300 \mathrm{~K}$ & 2.519 & 1.224 & 1.313 & 164.5 \\
$200 \mathrm{~K}$ & 2.534 & 1.141 & 1.401 & 167.9 \\
$20 \mathrm{~K}$ & 2.541 & 1.106 & 1.446 & 169.8 \\
\hline
\end{tabular}



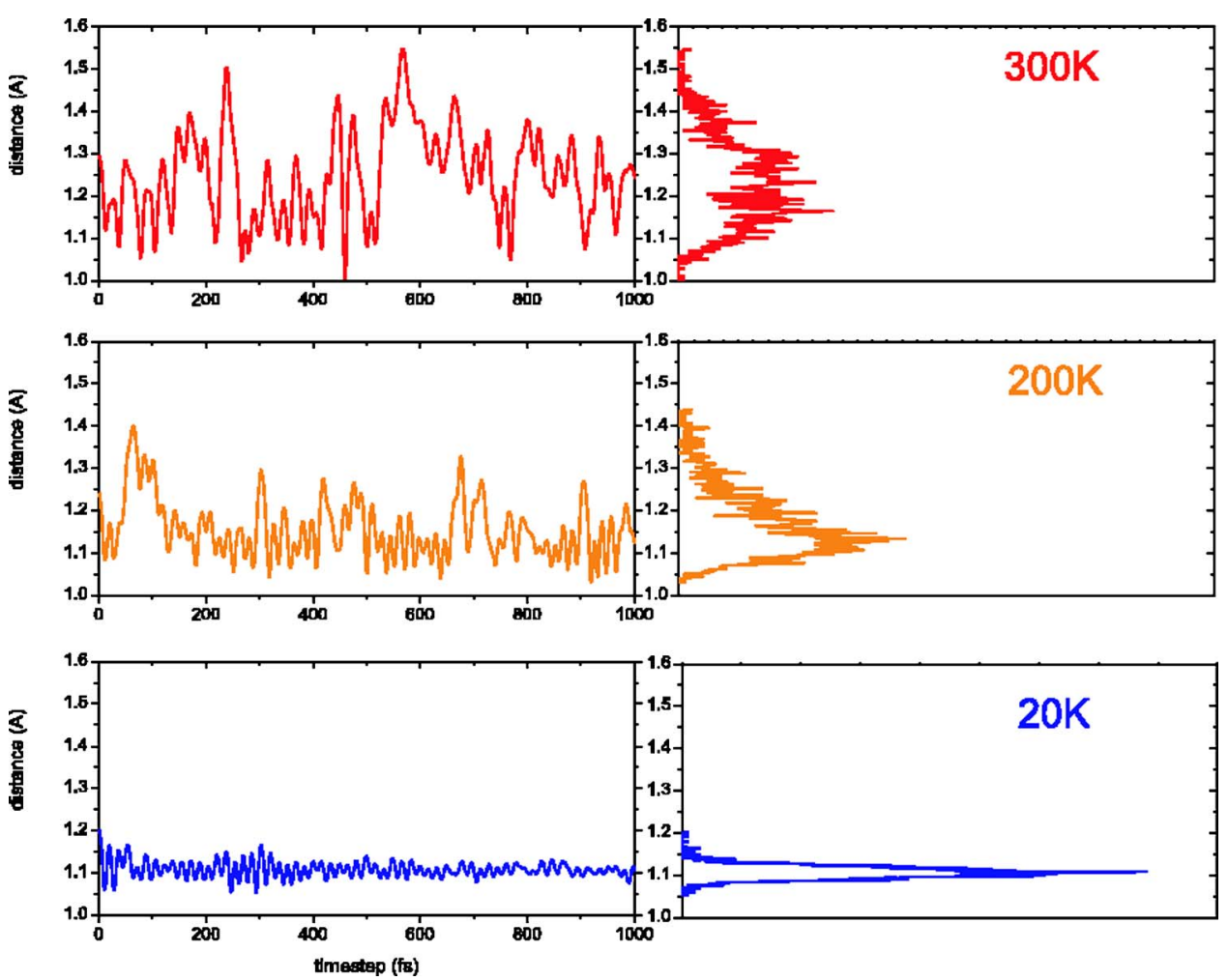

FIG. 10. Geometries obtained for the short hydrogen bond $\mathrm{N} 1 \cdots \mathrm{H} 1 \cdots \mathrm{O} 22$ in BTA-BPY by MD simulation at three temperatures. Histograms integrated over the full trajectories are shown on the right.

\section{DISCUSSION}

Using INS and $a b$ initio $\mathrm{MD}$, we have investigated three more hydrogen-bonded systems that show proton migration as a function of temperature. In these systems the SSHB are of the type $\mathrm{N}-\mathrm{H} \cdots \mathrm{O}$, compared to UPA which has an $\mathrm{O}-\mathrm{H} \cdots \mathrm{O}$ SSHB. Proton migration is therefore not chemically limited to a particular type of hydrogen bond.

Neutron diffraction provides the reference crystallographic data, which are the basis of all simulations presented here. As in the case of UPA, solid state DFT methods tend, in geometry optimization and low temperature MD calculations, to overestimate the donor-acceptor distance and underestimate the donor- $\mathrm{H}$ distance, which hinders the proton mi-

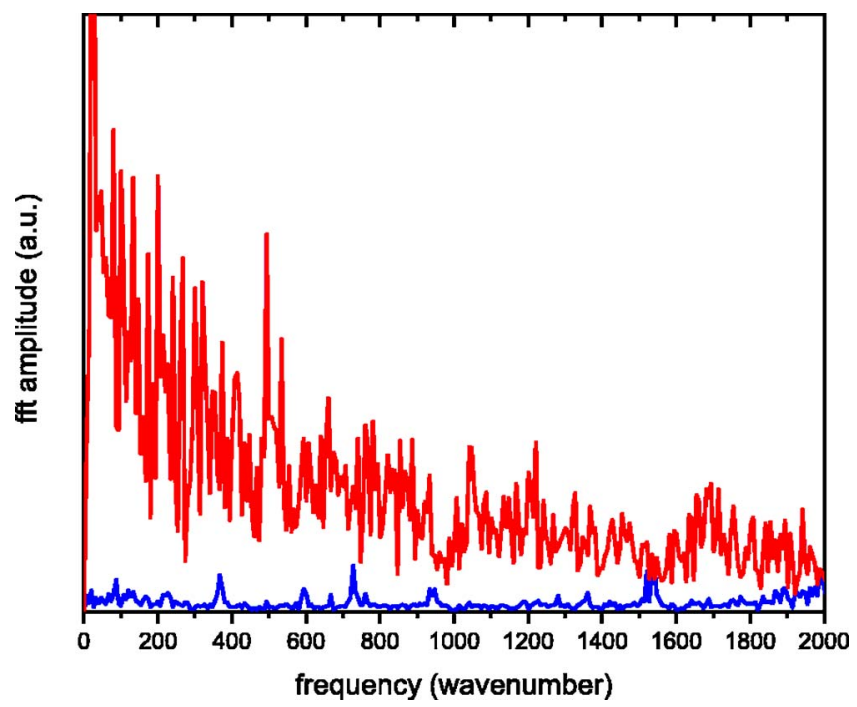

FIG. 11. The Fourier transform amplitude of the time-dependent N1-H1 bond length variation at $300 \mathrm{~K}$ (upper curve) and $15 \mathrm{~K}$ (lower curve). gration effect in the simulations. This trend is most pronounced in the case of 4MP-PCP to the extent that there is no evidence of proton migration in the MD simulations. In 4MP-PCP the hydrogen bonds connect the two molecules in dimers, whereas all other systems studied present infinite chains (BTA-BPY), 2D sheets (PDA), or 3D networks (UPA). Infinite networks of hydrogen bonds provide long range constraints that do not exist for a dimer, and in this case the SSHBs have more freedom to lengthen on geometry optimization.

In all other cases, the extent of proton migration in MD simulations is less than in the corresponding measurements. Increasing the temperature above that of the experiments helps to an extent but does not allow the full amplitude of

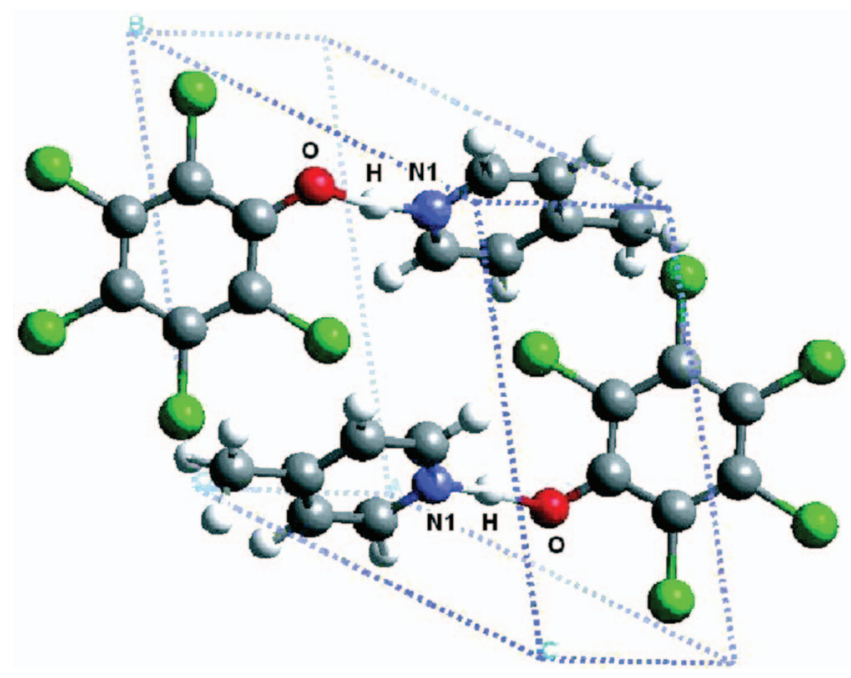

FIG. 12. (Color) Low temperature crystal structure of the 1:1 crystal of 2-methylpyridine and pentachlorophenol as reported in Ref. 11. 
TABLE V. Proton transfer as a function of temperature in the $\mathrm{N} 1 \cdots \mathrm{H} \cdots \mathrm{O}$ SSHB in 4-MP-PCP as reported in Ref. 11; experimental data and simulation results.

\begin{tabular}{lcccc}
\hline \hline & $\mathrm{N} 1 \cdots \mathrm{O}$ & $\mathrm{N} 1 \cdots \mathrm{H}$ & $\mathrm{H} \cdots \mathrm{O}$ & $\mathrm{N} 1-\mathrm{H}-\mathrm{O}$ \\
\hline Expt. data & & & & \\
$200 \mathrm{~K}$ & 2.525 & 1.304 & 1.229 & 170.6 \\
$100 \mathrm{~K}$ & 2.513 & 1.265 & 1.258 & 170.1 \\
$20 \mathrm{~K}$ & 2.506 & 1.206 & 1.309 & 170.4 \\
MD simulations & & & & \\
$300 \mathrm{~K}$ & 2.562 & 1.136 & 1.441 & 166.7 \\
$200 \mathrm{~K}$ & 2.561 & 1.112 & 1.462 & 167.8 \\
$20 \mathrm{~K}$ & 2.616 & 1.11 & 1.521 & 168.7 \\
\hline \hline
\end{tabular}

migration to be reproduced. In addition to the problems mentioned above of SSHB geometry, the fact that the protons are treated as classical, point particles instead of partially delocalized wave functions also hinders proton migration. Calculation of the eigenfunctions in the potential well of the proton along the donor-acceptor direction indicates that neglecting the zero point motion amounts to a shift of $\sim 0.03 \AA$ of the proton away from the center of the SSHB, compared to a transfer distance of $\sim 0.1 \AA$.

One significant difference between the $\mathrm{N}-\mathrm{H} \cdots \mathrm{O}$ SSHBs studied here and the $\mathrm{O}-\mathrm{H} \cdots \mathrm{O}$ SSHB in UPA is that, experimentally, the protons in all three cases migrate beyond the center of the hydrogen bond. In BTA-BPY evidence of this behavior is seen in the histogram of proton positions (Fig. 10) and the time-dependent traces of the $\mathrm{N}-\mathrm{H}$ distance which clearly show the proton spending a significant amount of time beyond the center of the hydrogen bond. This effect is much clearer in PDA. The time dependence of the $\mathrm{N}-\mathrm{H}$ distance shows almost periodic hopping of the proton from one side of the hydrogen bond to the other, and the histogram clearly shows two peaks separated by $\sim 0.1 \AA$, as in the experiment.

Fourier transforming the time domain information shows that, as in the case of UPA, low frequency lattice vibrations are driving proton migration. For BTA-BPY, the time dependence of the $\mathrm{N}-\mathrm{H}$ distance does not show any obvious modulation and there are no clear peaks in the FT. For PDA, there is almost periodic hopping across the center of the hydrogen bond (period 100-200 fs) and the FT (Fig. 7) shows an initial peak at low frequency $\left(<50 \mathrm{~cm}^{-1}\right)$ and two well-defined peaks at 115 and $145 \mathrm{~cm}^{-1}$. INS and phonon calculations show that these features correspond to vibrations in the plane of the molecular sheets which give rise to the strongest modulation of the hydrogen bond geometry.

Analyzing structures for PDA from the MD trajectories show that the lattice vibrations tend to stabilize the proton position on either side of the SSHB rather than in the center. The corresponding potential wells are single well and asymmetric for the offset proton positions and flat bottomed for the central position. In a model of a single particle oscillator of one proton mass, the calculated vibrational frequencies are lowest for the flat-bottomed potential with a limiting value of $1200 \mathrm{~cm}^{-1}$. These $\mathrm{N}-\mathrm{H}$ stretching movements, coupled in reality to other high frequency vibrations, can never play a role in proton migration which occurs at temperatures lower than $300 \mathrm{~K}$.

The apparent bistable behavior of SSHBs in which protons migrate beyond the center of the hydrogen bonds has not been detected experimentally. The time-dependent traces and histograms shown here are in the reference frame in which the donor $\mathrm{N}$ atom is stationary. Calculating the same quantities in the reference frame centered at the origin of the crystallographic cell gives rise to broad, featureless histograms. Diffraction measurements only have access to these broad distributions. Spectroscopic measurements would therefore be required to probe a two-site jump process as have been performed for quantum tunneling between the two wells of the potential of a normal hydrogen bond. ${ }^{28}$ Whereas these quasielastic measurements were performed with $\mu \mathrm{eV}$ resolution, that is, on the nanosecond time scale, only $\mathrm{meV}$ resolution would be required to detect the fast hopping process predicted here.

\section{CONCLUSION}

We have extended initial work on proton migration in SSHBs in UPA to three more systems which show proton transfer. If DFT methods allow hydrogen bond geometries to be accurately determined (two out of three cases studied here), then MD simulations show the same effect as seen in neutron diffraction experiments, albeit to a lesser extent. The two systems for which the simulations are successful reveal the same mechanism for proton migration, as in UPA; that is, lattice vibrations stabilize solid state structures at nonzero temperatures for which the proton is partially or completely transferred. When a proton transfers completely from donor to acceptor, as in PDA and BTA-BPY, a bistable regime exists at intermediate temperature where the proton avoids a relatively unstable, centered position. In the case of PDA, specific phonons which modulate the SSHB geometry are seen to be responsible for proton transfer.

While many systems have SSHBs, only a few show proton migration behavior. Understanding this type of behavior requires a precise knowledge of the low frequency dynamics of the system, which has been achieved here for PDA with INS experiments and phonon calculations. Having identified specific phonons that drive proton migration, it would be useful to characterize the lattice dynamics of SSHB systems that do not show proton migration. In view of the key role of dynamics in proton migration, predicting the activity of SSHBs in complex systems is clearly very challenging.

\section{ACKNOWLEDGMENTS}

We are grateful to Sasha Ivanov, Charlotte Broder, and Elinor Spencer for initial work on PDA, Ron Ghosh for setting up computational facilities at ILL, and Didier Richard for help with visualizing simulation results.

\footnotetext{
${ }^{1}$ W. W. Cleland and M. M. Kreevoy, Science 264, 1887 (1994).

${ }^{2}$ K. S. Kim, K. S. Oh, and J. Y. Lee, Proc. Natl. Acad. Sci. U.S.A. 97, $6373(2000)$

${ }^{3}$ B. A. Katz et al., J. Am. Chem. Soc. 124, 11657 (2002).

${ }^{4}$ S. Anderson, S. Crosson, and K. Moffat, Acta Crystallogr., Sect. D: Biol.
} 
Crystallogr. D60, 1008 (2004).

${ }^{5}$ P. A. Jones and D. Takai, Science 293, 1068 (2001).

${ }^{6}$ C. C. Wilson, Acta Crystallogr., Sect. B: Struct. Sci. B57, 435 (2001).

${ }^{7}$ C. C. Wilson and C. A. Morrison, Chem. Phys. Lett. 362, 85 (2002).

${ }^{8}$ J. A. Cowan, Ph.D. thesis, University of Durham, UK 2002.

${ }^{9}$ J. A. Cowan, J. A. K. Howard, J. McIntyre, S. M.-F. Lo, and I. D. Williams, Acta Crystallogr., Sect. B: Struct. Sci. B61, 724 (2005).

${ }^{10}$ J. A. Cowan, J. A. K. Howard, G. J. McIntyre, S. Lo, and I. D. Williams, Acta Crystallogr., Sect. B: Struct. Sci. B59, 794 (2003).

${ }^{11}$ T. Steiner, C. C. Wilson, and I. Majerz, Chem. Commun. (Cambridge) 14, 1231 (2000).

${ }^{12}$ F. Fontaine-Vive, M. R. Johnson, G. J. Kearley, J. A. K. Howard, and S. F. Parker, J. Am. Chem. Soc. 128, 2963 (2006).

${ }^{13} \mathrm{http}: / / \mathrm{www}$. ill.fr/YellowBook/IN1/

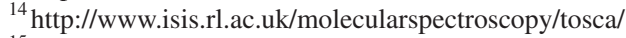

${ }^{15} \mathrm{http}: / / \mathrm{cms}$.mpi.univie.ac.at/vasp/

${ }^{16}$ G. Kresse and J. Furthmuller, Phys. Rev. B 54, 11169 (1996).

${ }^{17}$ G. Kresse and J. Furthmuller, Comput. Mater. Sci. 6, 15 (1996).

${ }^{18}$ G. Kresse and J. Hafner, Phys. Rev. B 47, 558 (1993).

${ }^{19}$ J. P. Perdew, K. Burke, and M. Ernzerhof, Phys. Rev. Lett. 77, 3685
(1996).

${ }^{20}$ G. J. Kearley, M. R. Johnson, M. Plazanet, and E. Suard, J. Chem. Phys. 115, 2614 (2001).

${ }^{21}$ M. Plazanet, N. Fukushima, M. R. Johnson, A. J. Horsewill, and H.-P. Trommsdorff, J. Chem. Phys. 115, 3241 (2001).

${ }^{22}$ M. Plazanet, N. Fukushima, and M. R. Johnson, Chem. Phys. 280, 53 (2002).

${ }^{23}$ M. R. Johnson and H.-P. Trommsdorff, Chem. Phys. Lett. 364, 34 (2002).

${ }^{24}$ M. R. Johnson, K. Parlinski, I. Natkaniec, and B. Hudson, Chem. Phys. 291, 53 (2003).

${ }^{25}$ M. Plazanet, F. Fontaine-Vive, K. H. Gardner, V. T. Forsyth, A. Ivanov, A. J. Ramirez-Cuesta, and M. R. Johnson, J. Am. Chem. Soc. 127, 6672 (2005).

${ }^{26}$ K. Parlinski, AIP Conf. Proc. 479, 121 (1999).

${ }^{27}$ T. Rog, K. Murzyn, K. Hinser, and G. Kneller, J. Comput. Chem. 24, 657 (2003).

${ }^{28}$ M. Neumann, D. F. Brougham, C. J. McGloin, M. R. Johnson, A. J. Horsewill, and H.-P. Trommsdorff, J. Chem. Phys. 109, 7300 (1997) 